\title{
La República de la Raza. Política indígena y brujería en el Chile del siglo XIX
}

Resumen: Este artículo propone una lectura política de la República de la Raza Indígena o la Recta Provincia: una organización que existió en la provincia de Chiloé [Chile] durante buena parte del siglo XIX hasta su desarticulación en 1880, cuando sus miembros, tachados de brujos, fueron denunciados y perseguidos por diversos homicidios y por asociación ilícita. El artículo muestra las peculiaridades de esta organización, destacando su rol político, sus vínculos con la antigua república de indios y sus pretensiones de inserción en el contexto liberal de la República de Chile. Por último, evidencia uno de los modos en que operó la raza en esta república sudamericana.

Palabras clave: mapuche, historia regional, indígenas, política, medicina.

\section{The Race Republic. Indigenous politics and witchcraft in the 19th-century Chile}

Abstract: This article proposes a political reading of the Republic of the Indigenous Race or Recta Provincia: an organization that existed in the province of Chiloé [Chile] during most of the 19th century until it was dismantled in 1880, when its members, suspected sorcerers, were denounced and persecuted, accused of various homicides and illicit associations. The article shows the peculiarities of this organization, highlighting its political role, its links with the former república de indios, and its pretensions of insertion in the liberal context of the Republic of Chile. Finally, the article shows one of the ways in which race operated in this South American republic.

Keywords: mapuche, regional history, indigenous, politics, medicine.

\section{A República da Raça. Política indígena e bruxaria no Chile do século XIX}

Resumo: Este artigo propõe uma leitura política da República da Raça Indígena ou a Recta Provincia: uma organização que existiu na província de Chiloé [Chile] durante a maior parte do século XIX até seu desmantelamento em 1880, quando seus membros considerados como bruxos foram denunciados e perseguidos por vários homicídios e por associação ilícita. 0 artigo tem por finalidade mostrar as peculiaridades desta organização, destacando seu papel político, seus vínculos com a antiga república de indios e suas pretensões de inserção no contexto liberal da República do Chile. Finalmente, este artigo pretende mostrar uma das maneiras pelas quais a raça operou nesta república sul-americana.

Palavras-chave: mapuche, história regional, indígena, política, medicina.

Cómo citar este artículo: Tomás Catepillan Tessi, "La República de la Raza. Política indígena y brujería en el Chile del siglo XIX”, Trashumante. Revista Americana de Historia Socia/13 [2019]: 84-107.

DOI: 10.17533/udea.trahs.n13a04

Fecha de recepción: 16 de marzo de 2018

Fecha de aprobación: 17 de julio de 2018

Tomás Catepillan Tessi: Doctor en Historia por El Colegio de México. Actualmente es profesor del Instituto de Historia de la Pontificia Universidad Católica de Valparaíso y miembro de la Comunidad de Historia Mapuche.

Correo electrónico: tomas.catepillan@gmail.com

- 


\title{
La República de la Raza. Política indígena y brujería en el Chile del siglo XIX
}

\author{
Tomás Catepillan Tessi
}

El 2 de mayo de 1856 el gobernador de Castro, en la provincia de Chiloé, Eenvió preso al "indígena Cosme Damián Antil" con destino a la capital de dicha provincia ubicada en los confines australes de lo que era Chile. Según las anotaciones del gobernador, Antil se titulaba a sí mismo "procurador general de la provincia”, y con ese título firmaba una serie de comunicaciones dirigidas "a los de su raza" y a "los mayores de la provincia", con lo que promovía, según la autoridad, ciertos desórdenes contra la República de Chile. El mismo gobernador aclaraba en su comunicación que aquellos "mayores" no eran otros que "los hechiceros como él, que los hay en los diversos distritos [...] con quienes siempre está en contacto para todos los actos de sus creencias ireligiosas, y superticiones diabólicas". ${ }^{\prime}$

No sabemos si esa fue la primera vez que Antil cayó preso. Sí sabemos que no fue la última: en 1864 nuevamente fue detenido por las autoridades provinciales, esta vez, acusado de conspirar contra la república y promover cierta sublevación indígena en Chiloé,Valdivia y la Araucanía. ${ }^{2}$ Oportunamente, la prensa del país del Mapocho (correspondiente al centro de Chile) lo llegaría a comparar con aquel francés que se hizo titular Rey de la Araucanía. ${ }^{3}$ Pero Antil no logró ninguna sublevación, ni en la Araucanía, ni en Valdivia, ni en Chiloé (aunque tampoco sabemos si era esto lo que buscaba). Lo que sí logró fue una sentencia que ordenaba su remisión a la casa de orates, por el tiempo necesario para que recobrara la cordura. ${ }^{4}$

* En el artículo retomo parte de mi tesis doctoral,Tomás Catepillan Tessi, "La 'provincia de Chile': construcción del Estado-nación en Chiloé, 1830-1880" (Tesis de doctorado en Historia, El Colegio de México, 2017). Agradezco las lecturas y comentarios de Diego Morales, Héctor Nahuelpan e Ignacio Álamos, sin los cuales este texto no habría llegado a ser lo que es. Asimismo, agradezco la lectura y comentarios de los evaluadores anónimos.

1. ANHC, Santiago, Fondo Intendencia de Chiloé, vol. 61, s.f.

2. Véase por ejemplo El Mercurio (Valparaíso) 14 de septiembre de 1864; ANHC, Santiago, Fondo Gobernación de Castro, vol. 2, s.f.

3. El Mercurio (Valparaíso) 29 de octubre de 1864.

4. "Sumario contra Cosme Damián Antil", Ancud, 21 de noviembre de 1864. ANHC, Santiago, 
Volveremos después sobre este personaje. Por ahora basta saber que al igual que con Antil, autodenominado "procurador general" y acusado de brujo, la locura fue una de las formas más efectivas con que los agentes de la República de Chile lidiaron con la "raza indígena" de Chiloé. La locura y la exotización fueron quizá los únicos medios posibles con que esta república se permitió nombrar lo indio fuera de la Araucanía histórica, territorio autónomo hasta la década de 1880. Esta forma de nombrar lo indio es la razón por la cual casi no existen estudios históricos sobre la relación entre el liberalismo y la población indígena que no perteneciera a la Araucanía histórica en el primer siglo de vida de la República de Chile, a diferencia de otras partes de América. ${ }^{5}$

Luego de la Independencia, temprano y por decreto, se eliminó en la República de Chile toda mención formal a las antiguas razas de la monarquía, del mismo modo en que se intentaron extinguir las tierras de indios, los alcaldes de indios, los procuradores de indios y, en fin, a la república de indios así como a todo lo que remitiera a aquellas corporaciones monárquicas que agrupaban a las poblaciones indígenas tanto en lo que era Chile como en las diversas unidades políticas de la América hispana. Pero nosotros sabemos que los hitos en que usualmente encuentran solaz los profesores de historia nacional no necesariamente suponen cambios radicales e inmediatos. Y en este artículo pretendo mostrarlo para el caso de la República de Chile, para lo cual me valgo de la historia regional, a la cual entraremos por un mirador inusual y extraño: una provincia periférica y un caso judicial contra una organización de brujos. Hemos de acudir a este mirador inusual y extraño porque en él se ponen de manifiesto los sesgos raciales de la República de Chile, las pretensiones políticas de la población indígena de Chiloé y los orígenes conjuntamente republicanos, monárquicos y tradicionales de sus prácticas políticas decimonónicas, aunque fueran percibidas exclusivamente como magia y como prácticas homicidas, "irreligiosas" y "diabólicas" al decir del gobernador de Castro.

De ahí que la idea de una "república de la raza”, cuya expresión hace parte del título de este artículo, nos sirve para nombrar a la organización usualmente asociada a los brujos de Chiloé, porque esta fue una de las denominaciones que le dieron sus miembros, ${ }^{6}$ y también nos sirve para hablar de la República de Chile.

Fondo Ministerio del Interior, vol. 358, s.f. También se publicó en El Mercurio (Valparaíso) 1 de diciembre de 1864.

5. Un panorama del liberalismo y sus discusiones decimonónicas en Chile en Iván Jaksić y Sol Serrano, "El gobierno y las libertades. La ruta del liberalismo chileno en el siglo XIX", Liberalismo y poder. Latinoamérica en el siglo XIX, eds. Iván Jaksić y Eduardo Posada Carbó (Santiago: Fondo de Cultura Económica, 2011) 207-244. Los trabajos sobre otras partes de América que han servido de guía e inspiración a este artículo son Florencia E. Mallon, Campesino y Nación. La construcción de México y Perú poscoloniales (México: Centro de Investigaciones y Estudios Superiores en Antropología Social / El Colegio de Michoacán / El Colegio de San Luis, 2003); Claudia Guarisco, La reconstitución del espacio político indígena. Lima y el Valle de México durante la crisis de la monarquía española (Castelló de la Plana: Publicacions de la Universitat Jaume I, 2011); María del Rocío Ortiz Herrera, "Elecciones y liberalismo en las Tierras Altas de Chiapas, 1863-1875", Ulúa 22 (2013): 103-133.

6. Esta organización también fue denominada Recta Provincia y La Mayoría. Véase "Copia de 
Una república liberal, pero no por eso menos organizada en torno a determinadas nociones de lo que eran las razas indígena y española, y alrededor del objetivo velado de asimilar la primera de estas.

Por otra parte, la idea de "política indígena" también encierra una valiosa ambivalencia. Usualmente, la expresión es utilizada para referirse a las políticas públicas que tratan sobre los indígenas, con lo que nuevamente los indígenas son categorizados como actores pasivos frente a un Estado activo. ${ }^{7}$ En cierto sentido, en este artículo hago referencia a esas "políticas indígenas" del Estado de Chile: sus pretensiones asimilacionistas, su pretendida "ceguera" frente a las diferencias raciales y su territorialización de lo indígena en la Araucanía.

En otro sentido, y el más importante para este texto, aquí hablo de "política indígena" para hacer referencia a las pretensiones y actividades políticas de la población indígena de Chiloé durante el siglo XIX. Aunque para hacerlo deba necesariamente salirme de los esquemas tradicionales que restringen la actividad política a las formas reconocidas por el mismo Estado. Así como debe abrirme a la posibilidad de estudiar, en las más diversas y a veces crípticas manifestaciones culturales, la organización política de un sector de la población, al cual se le vedó durante todo el siglo XIX la posibilidad de organizarse políticamente por fuera del estricto marco de la ciudadanía chilena. ${ }^{8}$

Ciertamente, la República de la Raza Indígena en Chiloé incorpora novedades respecto de la visión tradicional de la cultura mapuche, a la cual sin duda alguna deben vincularse los ritos, procedimientos médicos e incluso las expectativas de los brujos de Chiloé. Sin embargo, esto no vuelve a aquellos chilotes necesariamente "mestizos", o miembros de una cultura en decadencia o extinción. Mucho menos vuelve falsa la reivindicación indígena de aquellos habitantes de la Patagonia insular. En palabras de la profesora Paula López: "la autoctonía no antecede a las relaciones sociales ni a la historia, sino que es su resultado", , o, lo que es lo mismo, que la posición de alteridad, así como la reivindicación y los contenidos de cualquier identidad, proceden de interacciones sociales pesquisables. Podríamos decir que son históricas. ${ }^{10}$

algunas piezas del proceso de los brujos de Chiloé”, Ancud, 1880-1881. ANHC, Santiago, Fondo Varios, vol. 246.

7. Véase como ejemplo Guillaume Boccara e Ingrid Seguel-Boccara, "Políticas indígenas en Chile (siglos XIX y XX). De la asimilación al pluralismo (el caso mapuche)", Revista de Indias 59.217 (1999): 741-774.

8. Se puede leer una reflexión general sobre el tema, aunque centrada en el tiempo reciente, en el trabajo de Marisol de la Cadena, "Política indígena: un análisis más allá de "la política", World Anthropologies Network / Red de Antropologías del Mundo 4 (2009): 139-171.

9. Paula López Caballero, Indígenas de la nación. Etnografía histórica de la alteridad en México (Milpa Alta, siglos XVII-XXI) (México: Fondo de Cultura Económica, 2017) 31.

10. Se puede ahondar en esta perspectiva siguiendo los pasos de Stuart Hall y Michel Foucault bajo la guía de Eduardo Restrepo.Véase Eduardo Restrepo, Teorías contemporáneas de la etnicidad. Stuart Hall y Michel Foucault (Popayán: Editorial Universidad del Cauca, 2004). 
Sobre la base de estas premisas y temas, en este artículo nos concentraremos en un caso excepcional que nos servirá para estudiar la política indígena en el Chile decimonónico. Guía este trabajo la hipótesis general que la República de la Raza Indígena, u organización de brujos de Chiloé, si bien se basó en principios mapuches, fue una reacción a los cambios políticos del siglo XIX, inspirada tanto en el orden político de la monarquía y los derechos y saberes de la antigua república de indios como en los aprendizajes políticos realizados por la población indígena en las primeras décadas de la República de Chile. ${ }^{11}$ Comenzaremos el texto caracterizando a la República de Chile, el proceso de territorialización de los antiguos indios del reino de Chile y los desafios que supuso a este proceso la anexión de la provincia de Chiloé. Seguido de esta primera entrada, nos enfocaremos en la brujería chiloense: la literatura sobre el tema, el proceso de 1880, los elementos mapuches en la República de la Raza Indígena, las peculiaridades de esta organización y sus raíces monárquicas. Por último, nos detendremos en los posibles sentidos que podemos percibir en la acción de dichos chiloenses del siglo XIX, acusados y perseguidos como brujos en los mismos años en que la República de Chile se expandía arrebatando tierra a mapuches, peruanos y bolivianos.

\section{La raza liberal}

Hablar de razas en la actualidad parece un desvarío y ciertamente lo es, si pensamos en las razas como lo han hecho los supremacistas blancos, los nazis alemanes y demás alterados. De todos modos, el uso que hago de este concepto es más mundano y pertinente, históricamente hablando.

Para los años y el lugar que nos ocupan, en general desde fines del siglo XVIII a fines del siglo XIX en Chile, dificilmente podríamos proyectar sobre el término "raza" la definición que se hizo de ella en el siglo XX. En primer lugar, porque las nociones biológicas que permitieron la imaginación de las razas humanas como categorías taxonómicas asociadas a determinados comportamientos o a un mayor o menor progreso bien no se habían desarrollado, bien todavía no eran lo populares que llegarían a ser.

Por el contrario, quienes vulgarmente hablaban de raza en el Chile de 1820, por poner un caso, hacían referencia más bien al linaje y a la reputación o la calidad, ${ }^{12}$ aunque ya desde fines del siglo anterior la apariencia y el color comenzaban a ser

11. Las palabras de origen mapuche, cuando han sido castellanizadas, las utilizo siguiendo las normas gramaticales y ortográficas del español (y por lo mismo las pluralizo con 's', según corresponda), de ahí que hable de mapuches y de machis. Las palabras de origen mapuche, cuando no han sido castellanizadas, las escribo en cursivas y sigo las reglas gramaticales y ortográficas de dicho idioma (ajustado al alfabeto Raguileo).

12. Nicola Foote y René D. Harder Horts, eds., Military Struggle and Identity Formation in Latin America: Race, Nation, and Community During the Liberal Period (Gainesville: University Press of Florida, 2010) 19.Véase también Marisol de la Cadena, ed., Formaciones de indianidad.Articulaciones raciales, mestizaje y nación en América Latina (Popayán: Envión, 2007) 21-31. 
Figura 1. Ubicación de la Provincia de Chiloé, siglo XIX
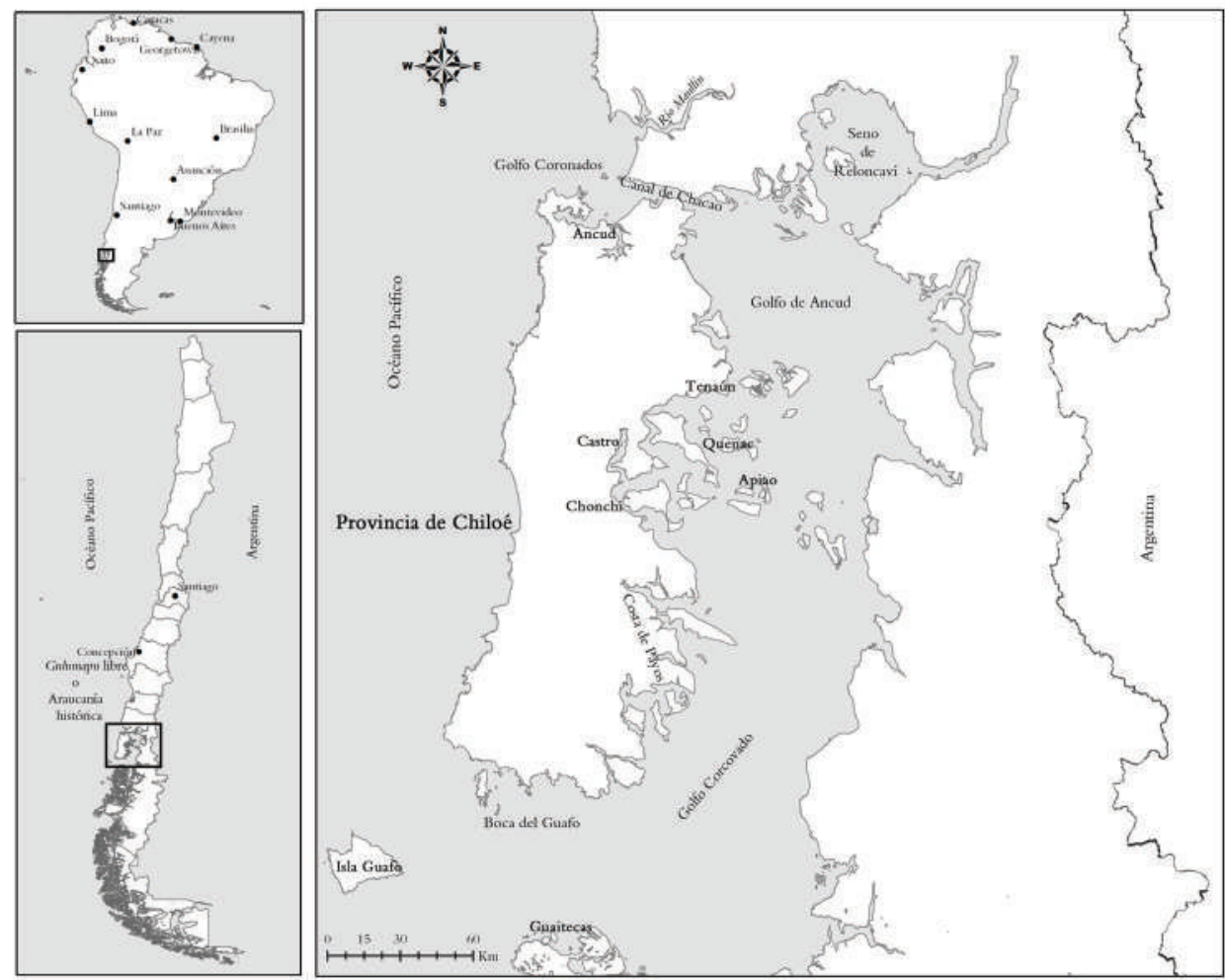

Fuente: Elaboración propia

elementos importantes en su caracterización. ${ }^{13}$ De ahí que el concepto de raza tuviera fuertes vínculos con determinados grupos locales —así es como se sigue usando en ciertas partes de México-y estuviera marcado por tener un contenido más jerárquico que biológico, en claro contraste con la imaginación de aquellas cuatro hipotéticas razas humanas. ${ }^{14}$

La principal distinción dibujada por la idea de raza en el reino de Chile, en consonancia con su uso jurídico, remitía a la imaginación de la sociedad como si estuviera dividida en dos grandes repúblicas: una de españoles y otra de indios.

13. Alejandra Araya Espinoza, "Registrar a la plebe o el color de las castas: 'calidad', 'clase' y 'casta' en la Matrícula de Alday (Chile, siglo XVIII)", América colonial. Denominaciones, clasificaciones e identidades, eds. Alejandra Araya Espinoza y Jaime Valenzuela Márquez (Santiago: RIL Editores, 2010) 331-361. También en De la Cadena, Formaciones 22-23.

14. Blanca, amarilla, negra y cobriza.Véase Tomás PérezVejo, "Raza y construcción nacional. México, 1810-1910", Raza y política en Hispanoamérica, coords. Tomás Pérez Vejo y Pablo Yankelevic (México: Bonilla Artigas Editores / El Colegio de México, 2017) 61-98. Sobre el carácter jurídico de la raza en Hispanoamérica a principios del siglo XIX véase Pérez 63, y sobre las cuatro razas humanas mencionadas véase Pérez 61. 
A este binarismo racial originario se terminó sumando un tercer conjunto compuesto por las castas y los esclavos o libertos de origen africano. Por último, entre los siglos XVIII y XIX, junto con la creciente importancia de la apariencia en la definición de la raza, se vivirá en Hispanoamérica una transición que hizo de la categoría "blanco" un reemplazo de la categoría "español”, como cúspide de la jerarquía social en estas partes del mundo. ${ }^{15}$

¿Qué rol pudo haber jugado este concepto de raza en la configuración de la República de Chile luego de las guerras civiles de 1810? Aparentemente, ninguno. Muy temprano, en 1818 y 1819, la autoridad chilena dispuso que los ciudadanos no debían denominarse españoles, sino chilenos, a pesar de que el término de antiguo se había usado para denominar a la población indígena. Asimismo, dispuso que los indígenas debían ser considerados ciudadanos sin distinción alguna. ${ }^{16}$

En parte como consecuencia de aquellas tempranas disposiciones que eliminaron la distinción entre indios, españoles y castas, que a diferencia de otros Estados americanos se mantuvieron consistentemente en las denominaciones y usos oficiales, ${ }^{17}$ en parte por la carga ideológica que irá adoptando el concepto de "raza", poco se ha hablado de esta categoría para caracterizar el proceso de construcción del Estado nación en Chile a lo largo del siglo XIX. Sin embargo, la raza fue fundamental para la imaginación de dicho Estado nación.

La eliminación formal de las razas en Chile facilitó la imaginación de una nación generalmente homogénea, compuesta de ciudadanos iguales, en contraste con población distinta, ubicada más allá de sus fronteras: los indios de la Araucanía histórica o el Gulumapu libre, cuya conquista termina recién en la década de 1880 , y en las fronteras septentrionales los bolivianos y peruanos, quienes habrían estado tocados por la africanidad y la indianidad según ciertos publicistas chilenos del siglo XIX. ${ }^{18}$

En otros términos, la matriz racial de la monarquía sirvió a los chilenos para relegar a los indios y a las castas fuera del territorio nacional. Esto supone, a su vez, que la ciudadanía chilena se montó sobre el vecinazgo y la raza española o blanca,

15. De la Cadena, Formaciones 24.

16. Jorge Pinto Rodríguez, La formación del Estado y la nación, y el pueblo mapuche. De la inclusión a la exclusión (Santiago: Dirección de Bibliotecas, Archivos y Museos, 2003) 72. Véase también el famoso decreto de Bernardo O’Higgins, "Sustitución de la denominación de 'español' por la de 'chileno'”, Santiago, 3 de junio de 1818. https://www.leychile.cl/Navegar?idNorma=1063811 \&idParte=\&idVersion $=1818-06-03(15 / 03 / 2018)$.

17. El caso contrario, por ejemplo, en Perú, Ecuador y Bolivia. A un primer momento en que se eliminan las distinciones raciales de la monarquía sigue el reconocimiento de dichas diferencias, e incluso la reactivación del tributo indio. Véase Brooke Larson, Indígenas, élites y Estado en la formación de las repúblicas andinas, 1850-1910 (Lima: Pontificia Universidad Católica del Perú / Instituto de Estudios Peruanos, 2002) 28-31.

18. Véase, por ejemplo, la tematización de la barbarie en la prensa chilena anterior a las guerras del Pacífico y de ocupación de la Araucanía, en Gabriel Cid, "De la Araucanía a Lima: los usos del concepto 'civilización' en la expansión del Estado chileno, 1855-1883”, Estudos Ibero-Americanos 38.2 (2012): 265-283. 
y que uno de los primeros y más importantes proyectos de la República de Chile fue la negación y asimilación de las antiguas razas dentro de su territorio bajo aquella ciudadanía española.

En otro lugar espero extenderme sobre las herramientas de la asimilación, algunas de las cuales fueron la categoría de "indio civilizado", la territorialización de los indios en la Araucanía histórica y aquel decreto de 10 de junio de 1823 que mandaba tasar y rematar las tierras de los pueblos de indios, de modo que se diluyeran en una estructura de propiedades individuales. Con todo, este proceso de asimilación no parece haber sido muy dificil en el país del Mapocho, aquel Chile central rápidamente controlado por la ciudad de Santiago: para principios del siglo XIX la población indígena de esta zona se encontraba en proceso de desaparición, los pueblos de indios despoblados, mal administrados y llenos de forasteros, sus tierras abandonadas y sus tributos apenas recolectados. ${ }^{19}$

Chiloé, anexado por Chile en el año 1826 luego de una exigente invasión, es otra cosa. ${ }^{20}$ Los censos y matrículas civiles y religiosas de fines del siglo XVIII señalan que la población de Chiloé se componía aproximadamente de un 50\% de población india y de un $50 \%$ de población española. ${ }^{21}$ Prácticamente no existían castas residiendo en la provincia y el binarismo racial de la monarquía se vivía con una rigidez importante: la categoría de mestizo no era utilizada por ningún habitante de Chiloé, en donde, no sé en qué grado con independencia de la calidad de los padres, los hijos eran indios o eran españoles. ${ }^{22}$

19. Sobre la situación de los pueblos de indios en el Chile a fines de la colonia véase Fernando Silva Vargas, Tierras y pueblos de indios en el Reino de Chile. Esquema histórico-jurídico (Santiago: Universidad Católica de Chile, 1962). Sobre los indios en los primeros años de la república véase Pinto, La formación. Lucrecia Enríquez, aunque se concentra exclusivamente en los años que van de 1810 a 1830, lo hace sin poner énfasis en los discursos de la raza, ni en la asimilación, ni en la identificación entre ciudadanía y raza española. Lucrecia Raquel Enríquez, "La república chilena ante la cuestión indígena (1810-1830)", Hispania Sacra 63.128 (2011): 627-652.

20. Sobre la anexión de Chiloé en 1826 véase Gonzalo Aravena Hermosilla, Chiloé 1826. El proceso de incorporación de Chiloé a la República de Chile, 1813-1831 (Castro: Ediciones 1826, 2017).

21. Véase, por ejemplo, el "Padrón general de la Provincia de Chiloé", San Carlos de Chiloé, 1785. ANHC, Santiago, Fondo Antiguo, vol. 26; "Plan general que demuestra el n de habitantes de la provincia de Chiloé con expresión de sus clases, estados y sexos", San Carlos de Chiloé, 1789. AGI, Sevilla, Gobierno, Indiferente General, legajo 1527; "Estado del colegio de Ocopa, y de todas sus misiones del Perú, y Chiloé”, Lima, 12 de octubre de 1791. AGI, Sevilla, Gobierno, Audiencia de Lima, legajo 1612.

22. Al respecto se pueden consultar los fondos con documentación colonial producida en la provincia de Chiloé (Archivo Nacional Histórico, Archivo General de Indias, Archivo General de la Nación del Perú). Véase también Rodolfo Urbina Burgos, Población indígena, encomienda y tributo en Chiloé: 1567-1813. Política estatal y criterios locales sobre el servicio personal de "veliches" y payos (Valparaíso: Pontificia Universidad Católica de Valparaíso, 2004) 64 y ss. Aunque este reconocido autor desdeña la ausencia del término "mestizo" en la documentación, utiliza ampliamente el concepto, que en sus términos habría sido "biológico y cultural", para dar cuenta del decaimiento de la población indígena. 
Más importante aún, la población india de Chiloé, que había sido incorporada tempranamente a la Monarquía Católica, tenía en su haber un largo proceso de evangelización, de sometimiento a la encomienda (si bien con algunos importantes levantamientos en su contra), de tributación al rey y, finalmente, de organización política indígena a partir de las últimas décadas del siglo XVIII, en concordancia con las normas establecidas en el virreinato del Perú para la república de indios y bajo la protección del Cabildo de Naturales de Lima. ${ }^{23}$

Más allá de las dificultades geográficas que supuso la anexión y control de Chiloé, en lo reseñado en los párrafos anteriores reside el principal desafio de aquella provincia austral a la construcción del Estado nación chileno. La política indígena chiloense no es más que una muestra de aquel desafio, aun cuando aquellos indígenas tuvieran por objetivo insertarse en la República de Chile sin renunciar a sus conocimientos y experiencias, ni mucho menos a sus identidades, como tendremos oportunidad de ver siguiendo el caso de los brujos chilotes de 1880 .

\section{El proceso judicial contra los brujos de Chiloé}

Se trata de una causa judicial que tuvo publicidad a los pocos días de iniciarse el proceso. La prensa de Ancud, capital de Chiloé, comenzó a informar del caso a principios de 1880, lo que coincidió con el inicio de las pesquisas y las detenciones ordenadas por el intendente de la provincia. No menos de 54 personas fueron encarceladas en el curso de la investigación, que duró prácticamente todo el año 1880 y algunos meses más. ${ }^{24}$

La documentación respecto de esta investigación, sin embargo, es precaria. Existen las sentencias de primera y segunda instancia, algunos artículos publicados en la prensa provincial, algunas órdenes y comunicaciones de las autoridades locales y, sobre todo, existe un documento titulado "Copia de algunas piezas del proceso de los brujos de Chiloé”. Se trata una selección de copias del proceso que mandó a sacar Ramón Espech, en 1882, para ser remitido a Benjamín Vicuña Mackenna. ${ }^{25}$

Tampoco es mucho lo que sabemos sobre el proceso judicial. Aparentemente, en algún momento entre febrero y marzo de 1880 el intendente de Chiloé ordenó que se detuviera en la provincia a todos los que tuvieran fama de brujos. ${ }^{26}$ Las órdenes de aprehensión se volverían a dictar en abril de 1880, en coincidencia temporal con las disposiciones militares que ameritaban la guerra en curso con Bolivia y Perú. ${ }^{27}$ Las detenciones, los interrogatorios y las pesquisas duraron todo el año. Para mayo de 1880 la principal autoridad de la provincia afirmaba que ya

23. Véase Catepillan, "La 'provincia de Chile"” 346-358.

24. La sentencia de primera instancia data del 2 de marzo de 1881. Véase "Sentencia de primera instancia contra Mateo Coñuecar y otros por asociación ilícita", Gaceta de los Tribunales (Santiago) 11 de junio de 1881.

25. Véase "Copia de algunas piezas del proceso" ff. 166r-166v.

26. "Copia de algunas piezas del proceso" f. 166.

27. "Decreto del intendente de Chiloé 7 de abril de 1880", El Chilote (Ancud) de 8 de abril de 1880; El Liberal (Ancud) 9 de abril de 1880. 
conocía el modo en que se organizaba aquella sociedad secreta de brujos y, más importante, que ya había puesto en retirada a dichos "médicos o hechiceros". ${ }^{28}$

A los detenidos se les acusó de diversos homicidios por envenenamiento y de asociación ilícita, siendo condenados por el juez letrado de la provincia solo nueve de ellos y exclusivamente por el delito de asociación ilícita. ${ }^{29} \mathrm{He}$ aquí que tenían constituida una "asociación secreta, compuesta en la generalidad de indígenas, y que tiene por objeto castigar a los que hacen mal, arreglado a sus leyes, que nos son enteramente desconocidas". ${ }^{30}$ Más todavía, según consta por la sentencia de primera instancia, resultó que esta sociedad se llamaba Recta Provincia, que sus miembros habían renombrado las principales localidades de la provincia, así como creado distritos, que tenían una jerarquía de autoridades, presidida por reyes y reinas y también presidentes, con sus supuestos intendentes, secretarios, comandantes, reparadores y médicos. Por último, constaba a la autoridad local que la gente acudía a ellos con la esperanza de averiguar los autores de determinados robos, de determinadas enfermedades e incluso de tal o cual muerte para que conforme la averiguación, los miembros de la Recta Provincia castigaran a los causantes de los daños denunciados. ${ }^{31}$ En segunda instancia la Corte de Apelaciones de Concepción revocó la primera sentencia y absolvió a todos los imputados: aquellos jueces consideraron que la organización no atentaba contra el orden social, las buenas costumbres, las personas o las propiedades, así como tampoco consideraron que la ley indígena en la que se basaba aquella organización contradecía la ley chilena. ${ }^{32}$

A partir del caso, y a pesar de su precariedad documental, se ha escrito bastante. Lamentablemente, estas escrituras en general reducen la brujería en el Chiloé de 1880 a una reliquia de tiempos pasados. En su defecto, interpretan a la Recta Provincia como una cultura tradicional en decadencia y perseguida por un Estado moderno, como una organización de resistencia al Estado y, alternativamente, como un suplemento de la precariedad de la administración judicial chilena. Por último, aquellas escrituras tratan la documentación del proceso judicial de 1880 como una de las principales fuentes para estudiar el folclor de Chiloé.

En los últimos años, de todos modos, han aparecido algunos textos en los que se nota un esfuerzo por entender la Recta Provincia en función de lo que pudo haber significado para sus miembros y adeptos. ${ }^{33}$ Sin embargo, todavía el debate

28. "Decreto del intendente de Chiloé 10 de mayo de 1880", El Chilote (Ancud) 13 de mayo de 1880; El Liberal (Ancud) 11 de mayo de 1880.

29. Véase "Sentencia de primera instancia contra Mateo Coñuecar". Mateo Coñuecar, de 70 años; Aurora Quinchen, de 40 años; Cristino Quinchen, de 61 años; Desiderio Quinchen, de 54 años; Domingo Coñuecar, de 54 años; Juan Ignacio Uribe, de entre 50 y 60 años; Juan Esteban Carimonei, de 26 años; Santiago Rain, de 98 años, y José María Chiguai, de 90 años, fueron condenados a penas de tres años.

30. Véase la vista fiscal firmada por José N. González. “Copia de algunas piezas del proceso” f. 199.

31. "Sentencia de primera instancia contra Mateo Coñuecar".

32. "Sentencia de segunda instancia contra Mateo Coñuecar y otros por asociación ilícita", Gaceta de los Tribunales (Santiago) 11 de junio de 1881.

33. Principalmente véase Fernando A. Valenzuela, "La enfermedad de todos en el cuerpo propio: 
sobre la Recta Provincia no ha logrado salir de su ensimismamiento, ni se ha conectado con los contextos provinciales y nacionales inmediatos al caso: la Guerra del Pacífico, el gobierno del intendente Luis Martiniano Rodríguez, la crisis económica de la década de 1870 — particularmente intensa en la provincia de Chiloé- una epidemia de viruela y la proliferación de ciertas partidas de bandidos, sobre todo en el extremo sur de la provincia. ${ }^{34}$ Mucho menos se ha conectado el debate con la historia de la población que organizó y sostuvo a la Recta Provincia: he aquí que esta organización perseguida en 1880, si bien se basaba en el azmapu (las normas consuetudinarias mapuches) y los principios de la etiología mapuche, también tenía elementos que muy probablemente fueron incorporados conscientemente por los políticos indígenas de Chiloé recién a principios del siglo XIX, y no huelga decirlo, de un origen asaz moderno y foráneo.

\section{Machi veliche, brujo chilote}

Empecemos por la parte tradicional de la organización de los brujos chilotes, es decir, por aquello que tiene de mapuche. Una parte que usualmente se ha prestado a equivocaciones. Lo primero que debemos aclarar es la denominación "veliche". En el Chiloé de principios del siglo XIX coexistían al menos tres identidades indígenas: reyunos de Calbuco, chonos australes asentados en la provincia por misioneros durante el siglo XVIII, y huilliches insulares, que fueron denominados como veliches durante el período colonial y parte del republicano. ${ }^{35}$ Aquellos veliches, cuyo nombre parece ser una corrupción española, ${ }^{36}$ para fines del siglo XVIII tenían una larga historia de separación con los huilliches continentales, e incluso con los segmentos mapuches septentrionales. Desde temprano fueron integrados

brujería y performatividad del tribunal de la raza indígena en Chiloé”, Universum 29.1 (2014): 35-55; aunque se centra en describir en términos abstractos el modo en que actuaba la Recta Provincia. Además, véase Joaquín Hernández Aracena, "Enfrentando saberes: los brujos de Chiloé y el discurso ilustrado (1849-1900)" (Tesis de maestría en Estudios Latinoamericanos, Universidad de Chile, 2013).

34. Véase Catepillan, "La "provincia de Chile"” 67-169.

35. Existe una cuarta categoría: "payos", que a mi parecer no denomina una identidad indígena. Se trata, más bien, de un término despectivo que utilizaban los españoles para designar a la población procedente de la costa suroriente de la Isla Grande de Chiloé. Esta región, una zona de contacto entre la población huilliche y la chona, bien pudo servir de hábitat a un conjunto de población distinta a la población indígena del norte de Chiloé. Sin embargo, no existe registro de ninguna palabra huilliche o chona que dé cuenta de esa diferencia. Más aún, en la actualidad, las principales y más antiguas agrupaciones huilliches se encuentran en la antigua zona de payos. El tema es interesante, aunque no del todo pertinente a este artículo. Se puede consultar Catepillan, "La "provincia de Chile"” 335-346.

36. Huilliches es castellanización de wijice, que significa "gente del sur". Que la voz veliche sea corrupción de wijice lo afirmó el jesuita Andrés Febrés, Arte de la lengua del reyno de Chile, con un diálogo chileno-hispano muy curioso: a que se añade la doctrina christiana, esto es, rezo, catecismo, coplas, confesionario, y pláticas, lo más en lengua chilena y castellana: y por fin un vocabulario hispano-chileno, y un calepino chileno-hispano más copioso (Lima: Imprenta de la Calle de la Encarnación, 1765) 3-4. 
a la monarquía: encomendados, evangelizados, masacrados cuando se resistieron y forzados a vivir en una parcial unión residencial con los españoles de la provincia de Chiloé. Es probable que esta separación entre huilliches continentales y huilliches insulares se acentuara por las recurrentes razias que realizaron los chilotes (españoles e indios) en tierras mapuches continentales durante los siglos XVII y XVIII, a despecho de que participaran de la misma cultura mapuche (compartían un mismo idioma, tenían ritos y una organización social análoga, etcétera). ${ }^{37}$

En este panorama geográfico e histórico, la pregunta recurrente sobre los brujos de Chiloé en 1880 se ha referido a la figura mapuche, que supuestamente encarnarían estos personajes. Mi posición, basada en etnografias contemporáneas, ${ }^{38}$ es que debemos comprender a los brujos como machis mapuches o, lo que es casi igual, machis veliches. ${ }^{39} \mathrm{Y}$ que a los machis podemos comprenderlos, en otros

37. Véase María Ximena Urbina Carrasco, La frontera de arriba en Chile colonial. Interacción hispanoindígena en el territorio entre Valdivia y Chiloé e imaginario de sus bordes geográficos, 1600-1800 (Santiago / Valparaíso: Dirección de Bibliotecas, Archivos y Museos / Ediciones Universitarias de Valparaíso / Pontificia Universidad Católica de Valparaíso, 2009).

38. Ana Mariella Bacigalupo, La voz del kultrun en la modernidad. Tradición y cambio en la terapéutica de siete machi mapuche (Santiago: Ediciones Universidad Católica de Chile, 2001); Ana Mariella Bacigalupo, Shamans of the Foye Tree. Gender, Power and Healing Among Chilean Mapuche (Austin: University of Texas Press, 2007); Rolf Foerster González, Introducción a la religiosidad mapuche (Santiago: Editorial Universitaria, 1995).

39. La discusión al respecto no está resuelta, más todavía existen varios autores que proponen que las antiguas machi de Chiloé pasaron a ser durante la colonia aquella figura de la evangelización que se llama fiscal de capilla, denominado en el idioma mapuche de la época como amomarikamañ. Entre esos autores se encuentra José Saavedra, Esteban Barruel y Luis Alberto Nahuelanca. De los tres solo Saavedra intenta explicar el montaje del fiscal de capilla sobre la antigua machi (aunque con una mala traducción del término amomarikamañ), los otros dos autores lo dan por sentado. Independiente de que puedan encontrarse elementos rituales mapuche en las celebraciones religiosas católicas de la provincia de Chiloé, me parece que en la evangelización de la provincia de Chiloé no predominó una tematización idolátrica, de modo que no hubo generalmente tendencias a montar los ritos católicos sobre los ritos locales (y mucho menos un montaje del fiscal de capilla sobre las machis). Al contrario, considerados como superstición, los ritos locales fueron más bien ignorados. Durante el siglo XIX esta ignorancia e incluso cierta condescendencia cambió: en el sínodo diocesano del obispado de Ancud de 1851 se consideró como grave pecado el curarse con machis, y más todavía se dispuso una grave sanción de vergüenza pública a los machis. El sínodo se puede consultar en Fernando Retamal, El primer sínodo chileno de la época republicana: Ancud 1851 (Santiago: Ediciones Universidad Católica de Chile, 1983). Otro argumento a favor de mi posición es que muy probablemente las antiguas machis limitaban su campo de acción a la sanación y el mantenimiento de la armonía. Para el caso de la Araucanía histórica, la inclusión de la machi en la realización de ritos como el gijatun (rogativa, rito principal mapuche que supuestamente perviviría en las fiestas patronales de Chiloé) se dará recién a fines del siglo XIX y como consecuencia del proceso reduccional impuesto por el Estado de Chile, de donde sería más probable pensar que el fiscal de capilla se hubiera montado (ien caso de haberlo hecho!) sobre otra figura mapuche como el genpin. Véase Foerster, Introducción 116-117; Miguel Melin y otros, Azmapu. Una aproximación al sistema normativo mapuche desde el rakizuam y el derecho propio (Santiago: Instituto Nacional de Derechos Humanos, 2016) 55. 
términos y utilizando una palabra muy querida por la antropología, como chamanes, aunque con algunas peculiaridades que anoto.

Hoy en día, generalmente, la machi es un personaje femenino, cuyo principal cometido es la sanación, la cual se realiza por medio de una refinada herbolaria, pero también a través de conocimientos místicos ya que la enfermedad en la cultura mapuche puede derivar de la acción de terceros, o incluso ser un castigo por faltas cometidas. Tanto en el caso de la acción de terceros como en el caso del castigo espiritual la enfermedad es vista como un desequilibrio, como el resultado de una transgresión del azmapu, o las normas consuetudinarias. ${ }^{40}$ La sanación que ejecuta la machi, por lo tanto, es concebida como la reparación del equilibrio en un grupo local y como el resguardo del azmapu.

La posibilidad que tiene toda machi de sanar, sin embargo, también las vuelve personajes ambiguos. En la misma medida que está cerca de lo numinoso y tiene un poder, la machi es también, y en potencia, un actor negativo causante del daño y el desorden, sobre todo, fuera del grupo que usualmente la reconoce en términos positivos: toda machi puede ser también un kalku, traducido desde antiguo como brujo en lo que tiene de negativo para la concepción occidental. ${ }^{41} \mathrm{Y}$ con este carácter ambivalente (positivo / negativo) de la machi, aparece un elemento central en las sociedades mapuches que fue reconocido desde la entrada de los españoles: su marcado carácter segmental. En términos de Rolf Foerster: "el admapu [...] es comprendido y apreciado como valores que se realizan en y por la propia comunidad discreta (ella se cree, de algún modo, 'representante de lo universal')", y esta comunidad "acepta que las otras comunidades lo realicen [el azmapu] bajo sus propias modalidades". ${ }^{42}$ De ahí que no existan machis antes del siglo XX que fueran reconocidas como autoridades espirituales de la totalidad del pueblo mapuche. Su autoridad ha estado tradicionalmente constreñida a lo local, a aquellas parcialidades denominadas rewe o cavi.

Lo que hacían los brujos chilotes de la época era precisamente lo que hacían sus contemporáneas machis en la Araucanía histórica (reparar el daño y resguardar el azmapu), aunque en un contexto bien distinto, con otro vocabulario y con pretensiones de diverso alcance que ciertamente parecen modernas. Impartían justicia, porque algunos vecinos no compartían sus víveres, ${ }^{43}$ por engaños entre

40. Azmapu significa literalmente semblante o costumbre de la tierra. Véase, por ejemplo, Tulio Cañumil y otros, Wixaleyiñ: mapucezugun-wigkazugun pici hemvlcijka. Pequeño diccionario castellanomapuche (Buenos Aires: El autor, 2008).

41. Véase Febrés, Arte de la lengua. Para Gonzalo Rojas, en cambio, machi y kalku son personajes contrarios. Según Rojas, los brujos de Chiloé serían kalku que habrían proliferado por la persecución de la que fueron objeto las machi, al punto de ser casi exterminadas por el español.Véase Gonzalo Rojas Flores, Reyes sobre la tierra. Brujería y chamanismo en una cultura insular. Chiloé entre los siglos XVIII y XX (Santiago: Editorial Biblioteca Americana / Universidad Andrés Bello, 2002).

42. Rolf Foerster González, “¿Pactos de sumisión o actos de rebelión? Una aproximación histórica y antropológica a los mapuches de la costa de Arauco, Chile" (Tesis de doctorado, Universiteit Leiden, 2004).

43. "Copia de algunas piezas del proceso" f. $177 \mathrm{v}$. 
parejas, ${ }^{44}$ pleitos entre vecinos, ${ }^{45}$ para vengar muertes (mágicas o mundanas), ${ }^{46}$ para descubrir quién enfermaba a un ser querido. ${ }^{47}$ En resumen, según declaró el "brujo" Juan Ignacio Uribe el 6 de abril de 1880, resultó que habían reconocido demandas de:

Ignacio Díaz porque decía que Pedro Chiguai le había robado una sabanilla y atribuía al mismo un pasquín que había recibido la noche antes en que lo amenazaban a muerte; Melchor Soto para que le den un médico que reconozca a su mujer que estaba enferma; Fernando Santana para que sepa el ladrón que le había robado un chancho y unas labijas de molina; Santiago Mayorga para que sepa el ladrón que le comió como veinte ovejas; Pedro Cárcamo para que alcance al brujo que le había aplicado un mal crónico que tenía en la nariz y en la garganta; Agustín Mella para que alcance a descubrir el brujo que le había envenenado o enfermado a su mujer, dejándola tullida; Rosa Vera para alcanzar a descubrir al brujo que le había enfermado una hija que tenía; Juan Ignacio Avendaño para el mismo objeto por la enfermedad de su mujer, y José Patricio Curiman con el mismo fin por enfermedad de su hermano. ${ }^{48}$

\section{La República de la Raza}

Por su vocabulario y por la ampliación de ámbitos que sancionaban a los brujos de Chiloé ya es común pensar en esta organización como tribunal. ${ }^{49}$ Ciertamente, parece tratarse de un tribunal, aunque en el centro de esta organización parajudicial estuviera cierta "ley indígena" que las autoridades chilenas desconocían, que por su nombre en mapuzugun podríamos denominar azmapu, y a pesar de que este conjunto normativo no necesariamente era el mismo y uno solo en Chiloé y la Araucanía histórica.

Más que la denominación de tribunal, sin embargo, parece haber existido entre los brujos de Chiloé la denominación de República de la Raza. ${ }^{50}$ Más todavía, la duplicación de distritos y localidades con las que supuestamente contaba la Recta Provincia, así como su jerarquía y multiplicidad de cargos, parece remitirnos más a una organización política que a una judicial, sin que esto nos haga olvidar la íntima conexión entre la autoridad política y la administración de justicia en el Antiguo

44. "Copia de algunas piezas del proceso" ff. 172, 183.

45. "Copia de algunas piezas del proceso" f. 173.

46. "Copia de algunas piezas del proceso" ff. 177, 180, 181, 199v, 200, 200v, 183, 189v.

47. "Copia de algunas piezas del proceso" ff. 186v, 192r, 192v.

48. "Copia de algunas piezas del proceso" ff. 185r-185v.

49. Incluso, algunos autores han acudido a la denominación de "tribunal de la raza" para hacer referencia a ella a pesar de que no existe documentación de la época que pruebe este uso por parte de sus miembros. Véase "Copia de algunas piezas del proceso". Me parece que es Fernando Valenzuela quien utiliza por primera vez la expresión "tribunal de la raza" como si fuera una denominación usada por los miembros de la Recta Provincia.

50. "Copia de algunas piezas del proceso" f. 192v, es la copia de un documento sin fecha dirigido a la Recta Provincia, firmado por Serafin Ojeda (residente en Huyar, departamento de Quinchao) 
Régimen. ${ }^{51}$ De aquel vínculo parece proceder parte importante de la forma y pretensiones de esta organización de brujos chilotes.

Aunque no lo hemos dicho hasta acá, esta organización parece haber tenido para 1880 un complejo organigrama de autoridades y empleados junto con una organización del territorio provincial, que replicaba la organización y nombres de las localidades de la provincia con nombres que solo conocían sus miembros.

Así, por ejemplo, la parte sur de la provincia usualmente conocida como Payos era denominada España en la Recta Provincia. Achao, por su parte, recibía el nombre de Buenos Aires, Quicaví el de Lima o Salamanca, etcétera. ${ }^{52}$ Más importante, según las declaraciones y la documentación incautada a los procesados en 1880, la Recta Provincia parecía estar organizada jerárquicamente a nivel provincial con sus reyes (a lo menos dos) también denominados presidentes, sus vicepresidentes, intendentes, diputados, secretarios, comandantes, reparadores, brujos menores, recaudadores, porteros, entre otros.

La interpretación general ha hecho de estos cargos el signo ineludible de que la Recta Provincia estaba organizada coherente y jerárquicamente. Asimismo, ciertos conflictos entre brujos, fueran o no autoridades de la Recta Provincia, así como la indeterminación de los distritos bajo el mando de cada brujo (o las atribuciones de las autoridades), e incluso disputas por la sucesión regia, han sido interpretados en función de una hipotética crisis de la Recta Provincia anterior al proceso de $1880 .{ }^{53}$ Es mi parecer, al contrario, que estas ambigüedades y contradicciones bien pueden haber sido parte inherente del ejercicio de la organización de brujos, y no solo porque no hayan pruebas de que alguna vez la Recta Provincia actuó jerárquica y coherentemente: es una probabilidad de lo más plausible que aquellas pretensiones de organización chocaran con el carácter segmental mapuche, quizá todavía latentes en las concepciones chilotas de lo que era el azmapu.

En otras palabras, como organización que incorporaba elementos tradicionales y modernos, y como organización de reciente data, es probable que todavía no se hubiera resuelto su transición hacia una institución totalmente afín al Estado de Chile. En otras palabras, que si bien administraba el azmapu, la Recta Provincia pretendía administrarlo rompiendo su marcado carácter segmental, ${ }^{54}$ con lo que

51. ¡Y aún en el primer siglo de la República de Chile! Piénsese en la administración local de justicia que dependía de los encargados del gobierno local: inspectores, subdelegados, gobernadores (dependientes del intendente), junto con municipales y juez de letras, que en Chiloé hubo uno solo durante la mayor parte del siglo XIX.

52. Estos nombres, con algunas contradicciones (como un mismo nombre para dos localidades distintas), se pueden ver dispersos en "Copia de algunas piezas del proceso". Rojas publicó una lista ordenada de estos dobles nombres. Véase Rojas 121.

53. Especialmente en la obra de Gonzalo Rojas Flores.

54. Agradezco al primer dictaminador sus aclaraciones al respecto: podríamos proyectar sobre prácticamente todo el Antiguo Régimen un funcionamiento segmental, por lo que la pretensión de universalidad de los procedimientos de la Recta Provincia (atendían a la población de toda la provincia, independiente de que se definieran como república de indígenas) podría ser interpretada como un carácter que los distinguía tanto de la sociedad mapuche tradicional como 
muy probablemente debió generar fricciones locales. Pero esta pretensión de romper el carácter segmental mapuche no es la única novedad de la Recta Provincia que vale la pena mencionar en la misma medida que nos puedan hablar de sus orígenes y pretensiones políticas.

La primera novedad que cabe mencionar es que los brujos de Chiloé procesados en 1880 hablaban generalmente el idioma español, oían demandas y administraban justicia en esta lengua, y más todavía no veían como algo común el uso del mapuzugun. ${ }^{55}$ Sin que nada de esto obstara a que se reconocieran como "indígenas", ni que calificaran a sus comunes como de "raza indígena".

La segunda novedad que cabe mencionar es que la Recta Provincia, a pesar de lo que usualmente se ha querido proyectar en esta organización, ni se pretendía como una organización de resistencia contra el Estado de Chile, o contra la colonización blanca, ni pretendía ser un reducto tradicional autónomo capaz de sustraer a sus miembros de la sujeción a la República de Chile a la que todos ellos se debían como ciudadanos de esa república. Sobre lo segundo, vale la pena recordar algunos casos que involucraron a los miembros de la Recta Provincia y a sus adeptos.

El primero de estos casos aparece sintetizado en un documento incautado a los reos de 1880, fechado en Quicavía (localidad de Chiloé) el 26 de julio de 1878. Este documento es una enérgica condena emitida por el presidente Domingo Coñuecar contra Domingo Nahuelhuen, quien por ese entonces había alcanzado fama por dirigir ciertos crímenes en el sur de la provincia. ${ }^{56}$ Coñuecar no solo se refería a Nahuelhuen como un "indigno salvaje" y una vergüenza para "nuestra raza indígena": lo calificaba de impertinente, malsufrido e incluso de débil. En lo que nos interesa, esta condena contra un paisano acusado de diversos asaltos y homicidios sirvió a Coñuecar para recordarle a sus supuestos subalternos que no debían tener ninguna disputa con los "jueces civiles y políticos”, que debían ser obedientes a las órdenes de esos funcionarios y velar por los desórdenes de "nuestra raza". ${ }^{57}$

de la sociedad de Antiguo Régimen.

55. En todo el proceso de los brujos de Chiloé existe una sola mención del idioma mapuzugun: Pedro Antonio Güichapane, de 25 años y natural de Castro, declaró haber visto al machi Benito Panichine poner unas piedras en un vaso con agua, acto seguido de lo cual "comenzó a romancear en veliche" fijándose en el vaso, con la esperanza de averiguar quién tenía enfermo al paisano Colipichun.Véase la declaración del 7 de abril de 1880. "Copia de algunas piezas del proceso" f. 192v.

56. El caso de Domingo Nahuelhuen, fusilado el 9 de junio de 1879, merece un artículo aparte. Se puede consultar Catepillan, "La "provincia de Chile"” 87-98. El documento citado en el párrafo se encuentra en "Copia de algunas piezas del proceso" f. 196v. Como la mayoría de los autores que han trabajado los brujos de Chiloé han utilizado cierta publicación de estas copias, han leído "Nahuelquen" donde debía decir "Nahuelhuen" (en el original: "Nauelguen”). Más todavía, al menos uno de esos autores (por ejemplo, Rojas 163) ha querido ver en ese falso "Nahuelquen" a un personaje de 1849 , nombrado Nahuelquin (!), del que diré algunas cosas en los siguientes párrafos.

57. "Copia de algunas piezas del proceso" ff. 196v-197r. El calificativo usado por Coñuecar es el 
El segundo de estos casos nos remonta en el tiempo a 1849. Sabemos de este por medio de una publicación en la prensa chilota, la sentencia judicial del caso y algunas breves comunicaciones locales. ${ }^{58}$ En resumen, según la sentencia judicial, los indios del distrito de Apiao acudieron en 1849 a los brujos Domingo Nahuelquin, Virginio Guichacalen y Magdalena Levicoy para que los defendiesen de los abusos a los que los sometían las autoridades chilenas del departamento de Quenac. La noche del 20 de noviembre de 1849 hubo una disputa entre los indios de Apiao y los brujos, porque los primeros se sentían engañados al no ver remediados sus males. Los brujos amenazaron con derramar una peste en la isla y, finalmente, la población local decidió ultimarlos ahogándolos en el mar. ${ }^{59}$ Según testimonio de un vecino de Quenac que dijo haber presenciado las declaraciones en 1849, los indígenas de Apiao habían acudido a Nahuelquin porque lo consideraban su rey y esperaban que les proveyera justicia, castigando a las autoridades chilenas. Y en efecto, Nahuelquin los contentó proclamando que sentenciaba a muerte al gobernador de Quenac, al párroco local y a dos vecinos. La sentencia, de todos modos, no se ejecutó. El cronista no nos explica por qué Nahuelquin mandó llamar al "Virrey de Lima", es decir de Quicaví, quien desestimó la sentencia, lo llamó "ladrón, embustero, fascineroso", amenazó con denunciar la situación a las autoridades y exigió ser devuelto a su hogar. ${ }^{60}$ Defraudados, nos dice el cronista, los indios de Apiao asesinaron a Nahuelquin y su compañía.

En este caso no hay nada tan claro como la recomendación de Coñuecar (¡no interfieran a las autoridades chilenas!), a pesar del conflicto opera la misma lógica: la ley indígena de la Recta Provincia no podía imponerse a la ley chilena, aunque fuera de justicia condenar a muerte a determinado gobernador local. Lamentablemente, no he podido encontrar más casos en los que las autoridades chilenas sean desafiadas por la población indígena de Chiloé y, a la vez, casos en los que algunos personeros de la Recta Provincia hayan ordenado la obediencia y la sumisión, aún fuera en defensa del azmapu. Si consideramos este radical reconocimiento de la República de Chile, no sorprende que varios de los procesados en 1880 como brujos hubieran hecho considerables aportes monetarios para los esfuerzos de la guerra en $1864 .{ }^{61}$

de "fétido". Véase 'fétido', 'hediendo' y 'hediondez' en Real Academia Española, Diccionario de la lengua castellana, t. 3 (Madrid: Imprenta de la Real Academia Española, 1732). Quizá el énfasis no estaba puesto en lo malsufrido como en lo lujurioso, que también parece haber sido atributo de la hediondez.

58. Prudencio Barrientos, "Horribles crímenes por los brujos de Quinchao", El Chilote (Ancud) 17 de junio de 1880; "Sentencia de la Corte Suprema contra Mariano Paillacar y otros por homicidio", Gaceta de los Tribunales (Santiago) 10 de septiembre de 1851; Notas de mayo a julio de 1850 del Gobernador de Quenac. ANHC, Santiago, Fondo Gobernación de Quenac, vol. 2.

59. Véase "Sentencia de la Corte Suprema contra Mariano Paillacar y otros por homicidio", Gaceta de los Tribunales (Santiago) 10 de septiembre de 1851.

60. Prudencio Barrientos, "Horribles crímenes por los brujos de Quinchao", El Chilote (Ancud) 17 de junio de 1880 .

61. "Erogaciones para el esfuerzo de la guerra”, Ancud, 8 de julio de 1864. ANHC, Santiago, Fondo 
¿De dónde podría proceder esta manera de comprender el resguardo de la ley indígena? Una manera de comprenderlo que quebraba el importante carácter segmental mapuche y del Antiguo Régimen, que obviaba el mapuzugun, y que no pretendía una autonomía radical, como en la Araucanía histórica y al estilo, por ejemplo, de Magiñ Wenu. ${ }^{62}$

Mi hipótesis, que ya he adelantado, es que la Recta Provincia fue una reacción innovadora producto de los cambios políticos del siglo XIX, y que para llevarse a cabo debió valerse de los aprendizajes realizados en las primeras décadas de la República de Chile, de la cultura política del período monárquico y, sobre todo, de los aprendizajes realizados por la población indígena en la antigua república de indios organizada en Chiloé a fines del siglo XVIII. ${ }^{63}$

En el caso del idioma, el asunto es decisivo. No tenemos espacio aquí para ahondar en la categoría de "indio civilizado" como una herramienta utilizada por la República de Chile para la asimilación. El indio neto, según esta categoría, no puede hablar el español. Caso muy distinto es el que encontramos a fines del siglo XVIII prácticamente en la totalidad de las dependencias americanas de la monarquía católica: los archivos están llenos de indios litigantes que no solo manejaban bien la lengua de Castilla, sino que además comprendían y se beneficiaban de la legislación indiana. ${ }^{64}$ Para aquellos indios de 1700, así como para los ciudadanos chilenos que formaron parte de la Recta Provincia, hablar castellano no era óbice para que se reivindicaran como indígenas, ni para que no fueran identificados como tales por sus contemporáneos.

Respecto a la relación entre la Recta Provincia y República de Chile, del mismo modo, el esquema parece darlo el carácter corporativo de la antigua república de indios, que por un lado reconocía a la autoridad real e incluso la preeminencia de las autoridades españolas en la provincia de Chiloé y, por el otro, asumía que eran solo ellos los que podían administrar la ley indígena, a partir de ciertos elementos tomados de la política monárquica y de la tradición mapuche, junto con nuevos elementos tomados de la política republicana, incluidas ciertas denominaciones.

Es un hecho que la república de indios en Chiloé se organizó recién en la segunda mitad del siglo XVIII, con consecuencias inmediatas en la vida de los

Ministerio de Guerra, vol. 467, n. 28. Por ejemplo, en Tenaún aparecen Domingo Coñuecar, Silvestre Runin y Miguel Raicahuin.

62. Magiñ Wenu fue un rico dirigente mapuche del siglo XIX. Le tocó vivir el período de las guerras civiles de 1810. Hasta su muerte, quizá en la década de 1860, receló de la República de Chile y se esforzó por combatirla, así como por limitar la entrada de los chilenos en tierras mapuches. Véanse los recuerdos de Juan Kajfukura y José Manuel Zúñiga en Tomás Guevara y Manuel Mañkelef, Kiñe mufü trokinche ñi piel. Historias de familias. Siglo XIX (Temuco / Santiago: Liwen / Editorial CoLibris, 2002).

63. Catepillan, "La 'provincia de Chile"” 346-358. Esto, entre otras cosas, determinó que en Chiloé la población indígena se reivindicara, hasta el siglo XX, con un genérico "indígena", "indio" o incluso "indígeno", despegado de etnónimos como huilliche, mapuche, veliche, etcétera.

64. Véase como ejemplo el trabajo de Guarisco. 
indígenas de aquella provincia. A este proceso, del cual fueron actores fundamentales los mismos indios de Chiloé, puede asociarse el término de la encomienda (en 1788), la designación de autoridades indígenas provinciales, la promoción del español como lengua franca, la reducción de los tributos y la exención parcial de los mismos. ${ }^{65}$

La prueba del vínculo entre la práctica política indígena durante la monarquía y durante la república se puede buscar en los archivos, y no creo que sea dificil encontrar casos que la ilustren. Que baste por ahora mencionar un documento bastante decidor: una solicitud del 4 de enero de 1835 firmada por Juan Levien, autodenominado "leal vasallo del Gobierno", hijo del antiguo cacique de Tranqui y dueño de ciertos potreros en el sur de la provincia. ${ }^{66}$ Más allá de lo llamativo que resulta el uso de formalidades monárquicas para dirigirse al gobierno de la República de Chile, Levien replicaba una estrategia que desde antiguo sirvió a su gente: reconocer la autoridad, acomodarse a sus intereses y luego negociar beneficios locales. Levien informó al gobierno de Chile que había extranjeros en los archipiélagos al sur de Chiloé con intenciones contrarias a la república. Acto seguido recordó a la autoridad que antiguamente no pagaba impuestos por sus potreros, y que quería seguir gozando de ese privilegio, además solicitó ayuda para expulsar de sus tierras a ciertos parientes suyos que se decían falsamente propietarios.

Como he dicho, me parece que es cosa de dedicar tiempo a los archivos para que aparezcan más casos que nos ilustren aquellas continuidades e innovaciones de la política indígena en Chiloé, entre la monarquía y la república. Quiero creer que el trabajo de archivo también podrá colaborar a estudiar de mejor modo el caso de la Recta Provincia. Por ahora, solo puedo ofrecer un personaje en cuya biografia podemos apreciar de manera clara mi hipótesis.

Cosme Damián Antil, con quien inicié este artículo, es el titular de la biografia a la que me refiero. Ya dijimos que a mediados del siglo XIX fue procesado por conspirar contra la república, considerado loco y remitido a la casa de orates. Este personaje, para dicha de nosotros, también figura en el proceso de 1880: alguno de los detenidos tenía en su poder una carta fechada el 23 de noviembre de 1866 escrita por Felipe Santiago Lebitureo, dirigida a Cosme Damián Antil. ${ }^{67}$ Pero más allá de la continuidad entre el grupo al que se dirigía Antil en la década de 1860 y los mayores y los brujos que fueron detenidos y procesados en 1880, me interesan sus afanes anteriores a la década de 1860.

65. Como mucho en la historia de Chiloé, este tema ya ha sido trabajado por Rodolfo Urbina, aunque a mi parecer yerra en su consideración de la agencia indígena para dar cuenta de este cambio, así como en las consecuencias que tendría para la provincia. El autor citado recurrentemente habla de decadencia, económica, política y moral (!), además desdeña la posibilidad de que los recurrentes caciques que viajaban a Santiago y Lima, o que escribían a las autoridades en Chile, Perú y Castilla, lo hicieran de motu proprio y no por influjo de los jesuitas, o algún otro español de doble corazón. Véase Urbina, Población indígena; Catepillan, "La 'provincia de Chile"”.

66. ANHC, Santiago, Fondo Ministerio del Interior, vol. 657.

67. "Copia de algunas piezas del proceso" ff. 197v-198r. 
Según el intendente de Chiloé, Antil había sido educado por el importante cacique Bernardo Huenchur, de quien había heredado el cacicazgo ${ }^{68}$ Perdió aquella dignidad cuando se abolió la distinción entre indígenas y españoles, y se ocupó en lo sucesivo como sacristán en una capilla de la provincia y como agricultor en tierras que le cedió el Gobierno chileno. ${ }^{69}$ Pero no solo eso, Antil, antes de haber sido preso en 1856 y 1864, fue electo municipal por el departamento de Castro en donde llegó, además, a oficiar como juez de primera instancia y a ser calificado como elector. ${ }^{70}$ En una oración: Antil fue cacique, machi, dirigente de la Recta Provincia, juez, elector y municipal chileno, sacristán y, aparentemente, también conspirador monarquista.

\section{Conclusión}

Si uno lo lee aislado, este esbozo de biografia no parece informarnos mucho. Ciertamente, nos cuesta trabajo imaginar a Cosme Damián Antil en la década de 1830, esforzándose por comprender la nueva política liberal, por insertarse en ella y, a la vez, ideando las formas en que podría resguardar su ley indígena, ¡su azmapu!, y las formas en que podría preservar la autonomía de su república de indios.

Sin embargo, visto en la perspectiva del caso contra los brujos de Chiloé en 1880 puede que Antil y los suyos sí hayan logrado algo de este preservar la autonomía de la ley indígena y de este reinventar la República de Indios durante los años que corren entre la anexión de Chiloé y la Guerra del Pacífico (1826-1880). Lo verdaderamente sorprendente es que lo hicieran en el contexto de la República de Chile: bajo su marcado liberalismo y su pretensión de una ciudadanía sin razas. En otras palabras, que lo hicieran bajo el imperio de una ciudadanía montada sobre la raza española y, por lo tanto, en paralelo a la relegación del indio en la Araucanía y a los esfuerzos por asimilar a la población indígena que vivía fuera de aquellos confines.

Como he dicho más arriba, es de estas operaciones de donde puede proceder la falta de estudios históricos en Chile sobre la relación entre el liberalismo decimonónico y las poblaciones indígenas, que por el contrario abundan en otras latitudes americanas. Y es a contrapelo de esta tendencia que he escrito este artículo,

68. Bernardo José Huenchur firmó, junto con otros distinguidos caciques, la solicitud que hicieron los indios de Chiloé al rey, en 1796, para que se les concediera la elección a pluralidad de votos de un corregidor y dos procuradores. Provincia de Chiloé, 4 de febrero de 1796. AGI, Sevilla, Gobierno, Audiencia de Chile, legajo 301

69. "Sumario contra Cosme Damián Antil".

70. "Listado de los ciudadanos electos para los cabildos de Chiloé en 1846", Ancud, 19 de mayo de 1846. ANHC, Santiago, Fondo Ministerio del Interior, vol. 197; "Lista de ciudadanos que han sido municipales y residen en el departamento de Castro", Castro, 22 de octubre de 1879. ANHC, Santiago, Fondo Gobernación de Castro, vol. 6, s.f.; “'Lista de los individuos calificados en el Departamento de Castro', sesión de la Cámara de Senadores 20 de marzo de 1843", Sesiones de los cuerpos lejislativos de la República de Chile, 1811 a 1845, t. 33, comp.Valentín Letelier (Santiago: Imprenta Cervantes, 1908). 
en primer lugar, gracias a las enormes posibilidades que nos ofrece la historia regional. En un país altamente centralizado como Chile, un procedimiento importante para hacer historia crítica del Estado nación puede ser, precisamente, mover el foco hacia los márgenes, porque es allí donde probablemente encontremos señas de aquellos procesos generales que en el centro han sido más o menos velados. Yo espero haber colaborado en la demostración de este hecho a través del artículo que estamos concluyendo, relativo a la política indígena en Chile y, por lo tanto, relativo a los discursos raciales que operaron en la configuración del Estado nación chileno. Los mismos discursos que hasta hoy habían logrado que viéramos a la Recta Provincia o República de la Raza (una organización política, racional y de pretensiones bien sensatas) como si hubiera sido exclusivamente un hato de anacrónicos, locos, mágicos y exóticos.

\section{Fuentes}

\section{Manuscritas}

Archivo General de Indias, Sevilla (AGI)

Fondo Gobierno

Archivo Nacional Histórico de Chile, Santiago (ANHC)

Fondo Intendencia de Chiloé

Fondo Antiguo

Fondo Varios

Fondo Gobernación de Castro

Fondo Gobernación de Quenac

Fondo Ministerio del Interior

Fondo Ministerio de Guerra

\section{Impresas}

Febrés, Andrés. Arte de la lengua del reyno de Chile, con un diálogo chileno-hispano muy curioso: a que se añade la doctrina christiana, esto es, rezo, catecismo, coplas, confesionario, y pláticas, lo más en lengua chilena y castellana: $y$ por fin un vocabulario hispano-chileno, y un calepino chileno-hispano más copioso. Lima: Imprenta de la Calle de la Encarnación, 1765.

Letelier, Valentín. Comp. Sesiones de los cuerpos lejislativos de la República de Chile, 1811 a 1845. Tomo 33. Santiago: Imprenta Cervantes, 1908.

Real Academia Española. Diccionario de la lengua castellana. Tomo 3. Madrid: Imprenta de la Real Academia Española, 1732.

\section{Periódicos y revistas}

El Mercurio (Valparaíso) 1864.

Gaceta de los Tribunales (Santiago) 1851, 1881. 
El Liberal (Ancud) 1880.

El Chilote (Ancud) 1880.

\section{Internet}

www.leychile.cl

\section{Bibliografía}

Aravena Hermosilla, Gonzalo. Chiloé 1826. El proceso de incorporación de Chiloé a la República de Chile, 1813-1831. Castro: Ediciones 1826, 2017.

Araya Espinoza, Alejandra. "Registrar a la plebe o el color de las castas: 'calidad', 'clase' y 'casta' en la Matrícula de Alday (Chile, siglo XVIII)”. América colonial. Denominaciones, clasificaciones e identidades. Eds. Alejandra Araya Espinoza y Jaime Valenzuela Márquez. Santiago: RIL Editores, 2010.

Bacigalupo, Ana Mariella. La voz del kultrun en la modernidad. Tradición y cambio en la terapéutica de siete machi mapuche. Santiago: Ediciones Universidad Católica de Chile, 2001.

. Shamans of the Foye Tree, Gender, Power and Healing Among Chilean Mapuche. Austin: University of Texas Press, 2007.

Boccara, Guillaume e Ingrid Seguel-Boccara. "Políticas indígenas en Chile (siglos XIX y XX). De la asimilación al pluralismo (el caso mapuche)". Revista de Indias 59.217 (1999): 741-774.

Cañumil, Tulio y otros. Wixaleyiñ: mapucezugun-wigkazugun pici hemvlcijka. Pequeño diccionario castellano-mapuche. Buenos Aires: El autor, 2008.

Catepillan Tessi, Tomás. "La 'provincia de Chile': construcción del Estado-nación en Chiloé, 1830-1880”. Tesis de doctorado en Historia, El Colegio de México, 2017.

Cid, Gabriel. "De la Araucanía a Lima: los usos del concepto ‘civilización’ en la expansión del Estado chileno, 1855-1883”. Estudos Ibero-Americanos 38.2 (2012): 265-283.

De la Cadena, Marisol. "Política indígena: un análisis más allá de 'la política"”. World Anthropologies Network / Red de Antropologías del Mundo 4 (2009): 139-171. . Ed. Formaciones de indianidad. Articulaciones raciales, mestizaje y nación en América Latina. Popayán: Envión, 2007.

Enríquez, Lucrecia Raquel."La república chilena ante la cuestión indígena (18101830)”. Hispania Sacra 63.128 (2011): 627-652.

Foerster González, Rolf. “¿Pactos de sumisión o actos de rebelión? Una aproximación histórica y antropológica a los mapuches de la costa de Arauco, Chile". Tesis de doctorado, Universiteit Leiden, 2004.

. Introducción a la religiosidad mapuche. Santiago: Editorial Universitaria, 1995. 
Foote, Nicola y René D. Harder Horts. Eds. Military Struggle and Identity Formation in Latin America: Race, Nation, and Community During the Liberal Period. Gainesville: University Press of Florida, 2010.

Guarisco, Claudia. La reconstitución del espacio político indígena. Lima y el Valle de México durante la crisis de la monarquía española. Castelló de la Plana: $\mathrm{Pu}-$ blicacions de la Universitat Jaume I, 2011.

Guevara, Tomás y Manuel Mañkelef. Kiñe mufü trokinche ñi piel. Historias de familias. Siglo XIX. Temuco / Santiago: Liwen / Editorial CoLibris, 2002.

Hernández Aracena, Joaquín. "Enfrentando saberes: los brujos de Chiloé y el discurso ilustrado (1849-1900)". Tesis de maestría en Estudios Latinoamericanos, Universidad de Chile, 2013.

Jaksic, Iván y Sol Serrano. "El gobierno y las libertades. La ruta del liberalismo chileno en el siglo XIX". Liberalismo y poder. Latinoamérica en el siglo XIX. Eds. Iván Jaksic y Eduardo Posada Carbó. Santiago: Fondo de Cultura Económica, 2011.

Larson, Brooke. Indígenas, élites y Estado en la formación de las repúblicas andinas, 1850-1910. Lima: Pontificia Universidad Católica del Perú / Instituto de Estudios Peruanos, 2002.

López Caballero, Paula. Indígenas de la nación. Etnografía histórica de la alteridad en México (Milpa Alta, siglos XVII-XXI). México: Fondo de Cultura Económica, 2017.

Mallon, Florencia E. Campesino y Nación. La construcción de México y Perú poscoloniales. México: Centro de Investigaciones y Estudios Sociales en Antropología Social / El Colegio de Michoacán / El Colegio de San Luis, 2003. Melin, Miguel y otros. Azmapu. Una aproximación al sistema normativo mapuche desde el rakizuam y el derecho propio. Santiago: Instituto Nacional de Derechos Humanos, 2016.

Ortiz Herrera, María del Rocío. "Elecciones y liberalismo en las Tierras Altas de Chiapas, 1863-1875”. Ulúa 22 (2013): 103-133.

Pérez Vejo, Tomás. "Raza y construcción nacional. México, 1810-1910”. Raza y política en Hispanoamérica. Coords. Tomás Pérez Vejo y Pablo Yankelevic. México: Bonilla Artigas Editores / El Colegio de México, 2017.

Pinto Rodríguez, Jorge. La formación del Estado y la nación, y el pueblo mapuche. De la inclusión a la exclusión. Santiago: Dirección de Bibliotecas, Archivos y Museos, 2003.

Restrepo, Eduardo. Teorías contemporáneas de la etnicidad. Stuart Hall y Michel Foucault. Popayán: Editorial Universidad del Cauca, 2004.

Retamal, Fernando. El primer sínodo chileno de la época republicana: Ancud 1851. Santiago: Ediciones Universidad Católica de Chile, 1983.

Rojas Flores, Gonzalo. Reyes sobre la tierra. Brujería y chamanismo en una cultura insular. Chiloé entre los siglos XVIII y XX. Santiago: Editorial Biblioteca Americana / Universidad Andrés Bello, 2002.

Silva Vargas, Fernando. Tierras y pueblos de indios en el Reino de Chile. Esquema 
histórico-jurídico. Santiago: Universidad Católica de Chile, 1962.

Urbina Carrasco, María Ximena. La frontera de arriba en Chile colonial. Interacción hispano-indígena en el territorio entre Valdivia y Chiloé e imaginario de sus bordes geográficos, 1600-1800. Santiago / Valparaíso: Dirección de Bibliotecas, Archivos y Museos / Universidad de Valparaíso, 2009.

Urbina Burgos, Rodolfo. Población indígena, encomienda y tributo en Chiloé: 15671813. Política estatal y criterios locales sobre el servicio personal de "veliches" y payos. Valparaíso: Pontificia Universidad Católica de Valparaíso, 2004.

Valenzuela, Fernando A. "La enfermedad de todos en el cuerpo propio: brujería y performatividad del tribunal de la raza indígena en Chiloé". Universum 29.1 (2014): 35-55. 